\title{
Transatlantica
}

Revue d'études américaines. American Studies Journal

\section{Vers une photographie des sentiments}

Le monde et sa performance par Lee Miller

\section{Elena Vaou}

\section{(2) OpenEdition}

Journals

Édition électronique

URL : https://journals.openedition.org/transatlantica/4375

DOI : 10.4000/transatlantica.4375

ISSN : $1765-2766$

Éditeur

Association française d'Etudes Américaines (AFEA)

Référence électronique

Elena Vaou, "Vers une photographie des sentiments », Transatlantica [En ligne], 1 | 2009, mis en ligne le 01 septembre 2009, consulté le 16 septembre 2021. URL : http://journals.openedition.org/

transatlantica/4375; DOI : https://doi.org/10.4000/transatlantica.4375

Ce document a été généré automatiquement le 16 septembre 2021.

\section{cc)}

Transatlantica - Revue d'études américaines est mise à disposition selon les termes de la licence Creative Commons Attribution - Pas d'Utilisation Commerciale - Pas de Modification 4.0 International. 


\section{Vers une photographie des sentiments}

Le monde et sa performance par Lee Miller

\section{Elena Vaou}

1 Quand elle photographie le monde, Lee Miller ne se soucie pas autant de son imitation que de sa mise en spectacle. C'est justement cette mise en scène permanente de son objet d'exposition - que cela soit la vue du désert égyptien à travers sa fenêtre, l'explosion d'une bombe nazie, ou sa propre image comme sujet de désir -, qui met en évidence les traits dominant sa vie aussi bien que son œuvre. Fascinée par l'objectif dès son plus jeune âge, alors que photographiée nue par son père, elle devient son fantasme interdit, Lee Miller semble envahie par l'ennui de la banalité du monde qu'elle arrangera à sa guise afin de le rendre moins commun. Lee Miller se distingue des photographes surréalistes davantage par son ardeur que par sa technique.

2 Découverts après sa mort dans le grenier de sa maison en Angleterre, soixante mille négatifs constituent aujourd'hui les "Archives Lee Miller »: ils donnent à voir les mouvements d'une femme qui tenta de rendre un peu plus imprévisible ce vingtième siècle qu'elle traversa. Une partie de ces œuvres sont exposées jusqu'au 4 janvier 2009 à la Galerie nationale du Jeu de Paume à Paris.

\section{Variations photographiques : les cinq vies d'une femme extraordinaire}

Lee Miller est née le 23 avril 1907 à Poughkeepsie, dans l'État de New York. Sa beauté ne laisse indifférent ni son père, photographe amateur, ni Horst P. Horst, George Hoyningen-Huene ou Edward Steichen, photographes de Vogue, qui font d'elle à l'âge de 20 ans un mannequin lumineux dans l'univers mondain de New York. Elle développe ainsi un rapport très physique avec la caméra, créant avec elle un échange érotique, 
libertin, spectaculaire, qu'elle exploitera amplement quelques années plus tard, quand elle passera de l'autre côté de l'objectif en devenant elle-même photographe.

4 Car, le côté ludique du "modeling " n'est qu'une étape qui lui permettra de découvrir le monde de la création artistique, un univers qu'elle explore durant son deuxième voyage à Paris, l'été 1929. Lee Miller décide de s'y installer et se construit alors un personnage exceptionnel, mettant en valeur les variations de sa personnalité. Elle rencontre Man Ray avec qui elle établit une relation aux facettes multiples : elle est son amante, sa muse, mais aussi celle qui développera quelques-unes de ses photos parfois les moins réussies - et les rendra remarquables. Elle devient alors également son rival. Aux côtés de Man Ray, elle s'initie non seulement aux techniques de la photographie dont la fameuse solarisation, mais également à son univers surréaliste. C'est ainsi qu'en 1931, Jean Cocteau la choisit pour incarner un triple rôle dans son film Le Sang d'un poète. Elle interprète à la fois les rôles de la bouche, de la sculpture, et du destin qui joue aux cartes, soit une triple figure aux traits rêveurs et esthétiques.

5 Lee Miller ne s'enfermera jamais dans un courant artistique, dans une ville, ou dans un mode de vie. Quand immanquablement, l'ennui s'installe, Lee Miller change de vie. Après un an avec Man Ray, elle rentre à New York. Elle s'associe à son frère Eric, également photographe, et collabore au magazine Creative Art, co-dirigé par Alfred Stieglitz, qui publie son propos: «Lee Miller est un puzzle surréaliste, ce qui, convenons-en, est en soi une énigme $»^{1}$ Elle oscille ainsi entre le monde de la mode, du théâtre et du cinéma, auprès des studios Warner Brothers. En 1933, elle prépare sa première exposition dans la galerie new-yorkaise de Julien Levy.

6 Son talent de photographe avant-gardiste est alors reconnu, mais pour l'insoumise qu'elle est, cela suffit pour lui donner de nouveau l'envie de tout quitter. Mariée à un riche fonctionnaire égyptien qu'elle rencontre à New York, Aziz Eloui Bey, elle embarque aussitôt pour Le Caire. Or pour Lee Miller, changement géographique est synonyme de changement de vie.

7 Elle passe trois ans en Egypte, entre 1934 et 1937. Elle joue le rôle d'épouse, pour bien vite lui substituer celui d'une photographe qui transcrit la culture et les paysages du pays de façon esthétique. Les influences surréalistes apparaissent dans le choix de la mise en scène de ses images. Elle regarde et fixe le désert comme un morceau de rêve, comme en témoigne son Portrait de l'espace (1937) : à travers sa caméra, la géographie même devient un espace - temps onirique qu'elle arrange à sa manière avant de l'immortaliser. Pari gagné avec l'Afrique : il est temps pour Lee Miller de changer de continent.

Durant un nouveau séjour à Paris au début de l'été 1937, Lee Miller rejoint l'avantgarde parisienne et rencontre son futur mari, le peintre surréaliste britannique Roland Penrose. Suite à un voyage en Roumanie, elle s'installe avec lui à Londres en 1939. Autrefois mannequin, muse, artiste, épouse/photographe en quête de rêve oriental, la Seconde Guerre Mondiale lui offre la possibilité d'explorer un domaine nouveau. En décembre 1942, elle obtient son accréditation de l'armée américaine pour Brogue, l'édition britannique de Vogue, et part aussitôt pour la Normandie. Elle réalise alors son premier reportage en tant que correspondante de guerre et poursuit sur les clichés de Saint-Malo (LIEN), de Paris (LIEN), ou de la campagne d'Alsace (LIEN), une fois ces régions libérées. Mais c'est surtout en juin 1945, avec les images qu'elle "tisse " à travers son objectif de la libération des camps de concentration de Buchenwald et Dachau, publiées dans l'édition américaine de Vogue, qu'elle marque les regards et les 
esprits. La cruauté enregistrée dans ses compositions en noir et blanc laisse entrevoir l'humanité des uns, l'inhumanité des autres, et la subjectivité des sentiments de celui qui se place dans l'espace limitrophe. "Germans are like this», "Believe it ${ }^{2}$ sont les titres sans tournures qu'elle choisit pour les articles qui accompagnent ses photographies de guerre.

9 À la fin de sa vie, Lee Miller avoue dans une interview ${ }^{3}$ que si elle devait revivre, elle ferait tout pour que ses œuvres ne masquent pas ses émotions, ne rationalisent pas ses sentiments. La racine de ce désir de fabriquer des émotions a sans doute trouvé son premier champ d'expression dans ses photographies de guerre. Son audace, immortalisée sur la pellicule par David E. Sherman - son bain pris dans la baignoire d'Hitler - n'est que la démonstration, non pas d'une façon de faire de l'art, mais d'une nécessité d'être, d'une façon totale de penser, de créer, d'exister à travers le contraste provocateur, la mise en scène des émotions et la distance absolue de tout sentimentalisme.

10 Son retour à Londres en 1946 coïncide avec le commencement d'une nouvelle vie. L'année suivante elle épouse Roland Penrose et donne naissance à leur fils Antony. Sa collaboration avec Brogue et le monde de la mode ne correspond plus à ses quêtes. De 1946 à 1953, son œuvre photographique se caractérise par deux travaux majeurs.

11 Le premier est la réalisation de portraits de Picasso, de Man Ray et de Tàpies qui accompagnent des biographies que Roland Penrose prépare pour leurs amis.

Le deuxième, elle le réalise à Farley Farm, dans le Sussex, où le couple s'installe et crée une sorte de village, un microcosme organique, constitué d'amis et d'artistes invités qui vivent et travaillent en communauté. Lee Miller publie dans Vogue un portfolio, une histoire composée de photographies prises à l'intérieur de ce monde particulier qu'elle a construit avec son époux et leurs complices, alors que ces derniers s'occupent des tâches ménagères et du jardinage.

13 Working Guests, titre de sa dernière création, est une compilation d'œuvres photographiques qui, malgré leur mise en scène, proposent une nouvelle façon de voir le monde: une vision incongrue de la banalité prise sur le vif, faite art, une vision fondée sur l'insignifiance. L'artiste et ses modèles fonctionnent dans un monde sans souci de reproduction de sens et fabriquent ensemble les images et les codes d'un spectacle visuel, portant son propre et unique mouvement. Le regard extérieur devient voyeur, s'introduisant dans un monde irreprésentable autrement qu'à travers l'œil de Lee Miller, co-énonciateur de l'événement photographié.

\section{L'exposition à Paris}

14 «L'Art de Lee Miller »: tel est le titre de l'exposition de 150 œuvres de Lee Miller, organisée par la Galerie nationale du Jeu de Paume à Paris du 21 octobre 2008 au 04 janvier 2009. Il s'agit d'une rétrospective organisée par le Victoria and Albert Museum qui regroupe pour la première fois une sélection de tirages originaux de l'artiste, conservés aux Lee Miller Archives, au Victoria and Albert Museum de Londres et dans de nombreuses collections internationales.

15 Une riche documentation et présentation biographique - complétée par des revues, des dessins, un collage, et un extrait du film de Jean Cocteau, Le Sang d'un poète-, initient le spectateur à un univers rempli de corps codifiés, de moments historiques en 
noir et blanc, de scénographies de mondes remplis d'émotions, passant de l'épouvante des crimes nazis à l'humour et à la légèreté de l'être.

Il faut mentionner ici l'intérêt tout à fait exceptionnel du documentaire projeté au sein de cette exposition, Lee Miller ou la traversée, film de Sylvain Roumette ${ }^{4}$. Il retrace la vie de Lee Miller, à partir de documents d'époque et des témoignages d'Antony Penrose et de David Scherman.

Soulignons ici un fait: ce n'est pas que pour sa technique photographique remarquable de maîtrise et d'innovation - qu'on aime le travail de Lee Miller. C'est surtout parce qu'à travers ses images, le spectateur acquiert une petite dose de liberté et est touché par cette grâce et cette variation vitale qui l'entraînent avec elles dans une spirale autant onirique que musicale.

C'est ainsi que ses photographies deviennent une source d'inspiration, nous communiquant l'énergie de vivre avec un peu plus d'audace, de devenir à notre tour artistes, les créateurs exceptionnels et privilégiés de notre propre vie.

\section{BIBLIOGRAPHIE}

CALVOCORESSI, Richard, Lee Miller: Portraits from a Life, London, Thames \& Hudson, 2005

BURKE, Carolyn, Lee Miller. Dans l'œil de l'Histoire, une photographe, trad. de l'anglais par MarieClaude Rideau, Paris, Éditions Autrement, 2007

HAWORTH-BOOTH, Mark, Lee Miller, ouvrage publié à l'occasion de l'exposition « L'art de Lee Miler » au Jeu de Paume, trad. de l'anglais par Jean-François Allain, Paris, Hazan, 2008

PENROSE, Antony, Les vies de Lee Miller, London, Thames \& Hudson, 2005

PENROSE, Antony, SCHERMAN, David E., Lee Miller's War, London, Thames \& Hudson, Paris, SARL, 20072005

PENROSE, Antony, WEENEY MAC, Alen, The Home of the Surrealists: Lee Miller, Roland Penrose and their Circle at Farley Farm, London, Frances Lincoln, 2008

\section{NOTES}

1. Creative Art, New York, mai 1933.

2. «Ainsi sont les Allemands ", «Croyez-le ». Vogue, New York, juin 1945. Voir « Lee Miller dans Vogue, ou les horreurs de la guerre »

3. In Lee Miller ou la traversée du miroir, film de Sylvain Roumette. France, 1995, 54 mn, Production Terra Luna Films.

4. France, 1995, $54 \mathrm{mn}$, Production Terra Luna Films. 
INDEX

Thèmes : Trans'Arts

\section{AUTEUR}

ELENA VAOU

Docteur en Histoire et Sémiologie de l'Image, Université Paris VII - Denis Diderot, Centre Louis Gernet 